\title{
Modelo sintético de traqueia para a realização de traqueostomia e cricotireoidostomia: melhorando as opções de treinamento com alternativa de baixo custo para o ensino na graduação médica
}

\author{
Synthetic tracheal model for tracheostomy and cricothyroidostomy: improving \\ training options with a low-cost alternative for undergraduate medical education
}

\author{
Natássia Alberici Anselmo', Kênia Maria Jordão Cazon², Amely Covalero da Silva Pinto \\ Antonio Carlos Morale Guerra Júnior ${ }^{4}$, Carlos Dario da Silva Costa ${ }^{5}$, Raphael Raphe ${ }^{6}$
}

\begin{abstract}
Anselmo NA, Cazon KMJ, Pinto ACS, Guerra Júnior ACM, Costa CDS, Raphe R. Modelo sintético de traqueia para a realização de traqueostomia e cricotireoidostomia: melhorando as opções de treinamento com alternativa de baixo custo para o ensino na graduação médica / Synthetic tracheal model for tracheostomy and cricothyroidostomy: improving training options with a low-cost alternative for undergraduate medical education. Rev Med (São Paulo). 2018 jan.-fev.;97(1):24-9.
\end{abstract}

RESUMO: Modelos experimentais sintéticos têm sido aplicados em diversas áreas de treinamento das habilidades. Nesse sentido, foi desenvolvido um modelo para treinamento de traqueostomia e cricotireoidostomia, reutilizável por até quatro vezes, com custo baixo (cinco reais), montado em tempo curto (cerca de 15 minutos). Trinta e sete alunos de um curso de medicina realizaram os procedimentos com o modelo sintético criado e, na sequência, opinaram anonimamente sobre sua eficácia, vantagens e desvantagens em relação ao treino de habilidades, por meio de um questionário semiestruturado. Como resultados, mais de $70 \%$ dos estudantes declararam que essa seria a melhor maneira de complementar o aprendizado das técnicas cirúrgicas e que o maior benefício se deu pela possibilidade de praticar diversas vezes o procedimento, visto que a aquisição de uma habilidade só pode ser obtida com de exaustivo treinamento. Entretanto,
$40 \%$ dos alunos que responderam o questionário apontaram as dificuldades do material sintético, principalmente com relação à sua pouca maleabilidade. Vale observar também que o material de treinamento biológico possui muitas desvantagens, como durabilidade, dificuldade de armazenamento, alto custo, não ser reutilizável e o fato de as normas de sociedades protetoras dos animais estarem cada vez mais rigorosas. Concluindo, modelos de baixo custo que são reutilizáveis e o mais fidedigno possível à realidade, como o deste estudo, demonstram a sua importância na disciplina de habilidades como uma alternativa para complementar o aprendizado da técnica.

Descritores: Desenvolvimento experimental; Traqueostomia/ educação; Tecnologia de baixo custo; Materiais de ensino; Estudantes de medicina; Educação médica; Ensino; Treinamento por simulação/métodos.

Trabalho apresentado no III Congresso da Associação Brasileira das Ligas Acadêmicas de Cirurgia, São Paulo, SP, 2017.

1. Acadêmica do Curso de Medicina da Faculdade Ceres - FACERES, São José do Rio Preto, SP. Email: natassiaalberici@hotmail.com; https://orcid. org/0000-0003-1004-6769.

2. Acadêmica do Curso de Medicina da Faculdade Ceres - FACERES, São José do Rio Preto, SP. Email: kenia_cazon@hotmail.com; https://orcid. org/0000-0002-6773-045X.

3. Acadêmica do Curso de Medicina da Faculdade Ceres - FACERES, São José do Rio Preto, SP. Email: amelycovalero@hotmail.com; https://orcid. org/0000-0002-8417-1717.

4. Acadêmico do Curso de Medicina da Faculdade Ceres - FACERES, São José do Rio Preto, SP. Email: acmgjunior@gmail.com; https://orcid.org/00000002-0073-9943.

5. Professor de Cirurgia do Curso de Medicina da Faculdade Ceres - FACERES, São José do Rio Preto, SP. Email: carlosdariocosta@hotmail.com; https://orcid.org/0000-0003-4115-1844.

6. Professor de Cirurgia do Curso de Medicina da Faculdade Ceres - FACERES, São José do Rio Preto, SP. Email: raphael@raphe.com.br; https://orcid. org/0000-0002-9253-2105.

Endereço para correspondência: Natássia Alberici Anselmo - FACERES. Avenida Anísio Haddad, 6751. CEP 15090-305 - São José do Rio Preto, SP, BR. 
ABSTRACT: Synthetic experimental models have been applied in several areas of skill training. In this sense, a model for tracheostomy and cricothyroidostomy training was developed, reusable up to four times, with a 5.0 reais cost, mounted in 15 minutes. Thirty-seven medical students performed the procedures and anonymously opined about the effectiveness of the model in relation to the training of the skills through a questionnaire, which addressed mainly advantages and disadvantages. As a result, more than $70 \%$ of the students declared that this would be the best way to complement the learning of the surgical techniques and that the greatest benefit was the possibility of practicing the procedure several times, since the acquisition of a skill can only be obtained through exhaustive training. However,

\section{INTRODUÇÃO}

$\mathrm{M}$ odelos experimentais sintéticos têm sido aplicados em diversas áreas de treinamento das habilidades médicas e cirúrgicas, em substituição ou concomitantes ao modelo animal ${ }^{1}$.

A traqueostomia é um procedimento cirúrgico, de caráter eletivo e, excepcionalmente emergencial, realizado através de uma abertura na parede anterior da traquéia, com a finalidade de estabelecer uma comunicação entre a traquéia e o meio externo, possibilitando assim a livre passagem de ar. As principais indicações são permitir ventilação mecânica em intubações orotraqueais prolongadas e obstrução aguda de vias aéreas. É uma medida extremamente necessária em muitos casos, além de ser uma via aérea mais segura, mais fácil de ser retirada e recolocada do que a cânula de intubação orotraqueal, não aumentando a incidência de pneumonias ${ }^{2,3}$. A cricotireoidostomia é um procedimento cirúrgico de técnica relativamente simples, podendo ser realizada por via cirúrgica ou por punção. Consiste na abertura da membrana cricotireóidea e estabelecimento de comunicação com o meio externo, promovendo o controle da via aérea. É utilizada preferencialmente em vítimas de trauma e em pacientes com insuficiência respiratória aguda grave em risco iminente de morte, na falha de outras técnicas menos invasivas, sendo preferivel em comparação à traqueostomia de emergência, visto que a membrana cricotireóidea é próxima à superfície cutânea, sendo necessária menor dissecção, evitando lesões de estruturas mediastinais, parede posterior da traqueia e esôfago.

O aluno da graduação médica inserido em modelos de ensino que usam exclusivamente peças animais costuma carecer de treinamento para o aperfeiçoamento da técnica, para o desenvolvimento das habilidades imprescindíveis à execução da traqueostomia e cricotireoidostomia, além da demonstrar falta de confiança necessária para a realização dos procedimentos de maneira segura e eficiente. No entanto, não é ético que apenas aprimorem seu aprendizado prático utilizando o ser humano como ferramenta ${ }^{4}$.
$40 \%$ of the students who answered the questionnaire pointed out the difficulties found in the synthetic material, mainly in relation to its low malleability. Even so, biological training material has many disadvantages such as durability, storage difficulty, high cost, not being reusable, and the fact that animal welfare standards are increasingly stringent. In conclusion, low cost reusable models, as reliable as possible, such as the one in this study, demonstrate their importance within the skills discipline as an alternative to complement the learning of the technique.

Keywords: Experimental development; Tracheostomy/ educação; Low cost technology; Teaching materials; Sudents, medical; Education, medical; Teaching; Simulation training/ method.

Seguindo a tendência mundial de investir em modelos alternativos para o ensino e respeitando os Princípios Humanitários da Experimentação Animal, que preza pelo "replacement" (substituição, na medida do possível, por métodos que não envolvam animais), este modelo é voltado para o treino de técnica e habilidade.

\section{MATERIAL E MÉTODOS}

Um novo modelo sintético de traquéia foi desenvolvido por acadêmicos da Liga Acadêmica de Cirurgia Geral da Medicina FACERES. Utilizou-se uma traqueia sanfonada de 15 centímetros, sendo a extremidade superior fechada com uma rolha (Figura 2), enquanto a inferior, fechada com uma bexiga. Um anel da traqueia foi colocado três centímetros abaixo da cartilagem tireóidea, de maneira que ficasse sobreelevada para simular a cartilagem cricoidea. Entre as cartilagens tireoidea e cricoidea, foi feita uma abertura de $1,5 \mathrm{~cm} \times 1,5 \mathrm{~cm}$ na face anterior da traqueia e envolta com fita crepe, para simular a membrana cricotireoidea, local de realização da cricotireoidostomia. Um retângulo cortado de garrafa pet de $5 \mathrm{~cm} \mathrm{x} 4 \mathrm{~cm}$ (Figura 2), foi usado para simular a cartilagem tireóidea e fixada com uma espuma por cima da traqueia sanfonada, logo abaixo da extremidade superior (Figura 3). Abaixo da cartilagem cricoidea, foi fixado uma espuma $(10 \mathrm{~cm} \times 8 \mathrm{~cm})$, a fim de simular os músculos pré-laríngeos e, por cima de toda a estrutura, uma pele artificial, de $18 \mathrm{~cm} \times 10 \mathrm{~cm}$ (Figura 4).

Acadêmicos que já passaram pela disciplina de Habilidades Cirúrgicas e integrantes da Liga Acadêmica de Cirurgia Geral foram convidados para participar do estudo (Projeto aprovado pelo Comitê de Ética em Pesquisa - CEP da FACERES sob no 2.240.024) e realizaram o treinamento em peças animais e no modelo proposto neste artigo, com o intuito de avaliar a efetividade do modelo com relação ao treinamento das técnicas de traqueostomia e cricotireoidostomia. Após o treinamento, foi solicitado aos alunos participantes que respondessem ao questionário semiestruturado para verificar suas impressões em relação ao modelo proposto, para posterior melhorias aplicáveis, caso necessário, e para verificar se o modelo pode atender ao objetivo deste trabalho. 


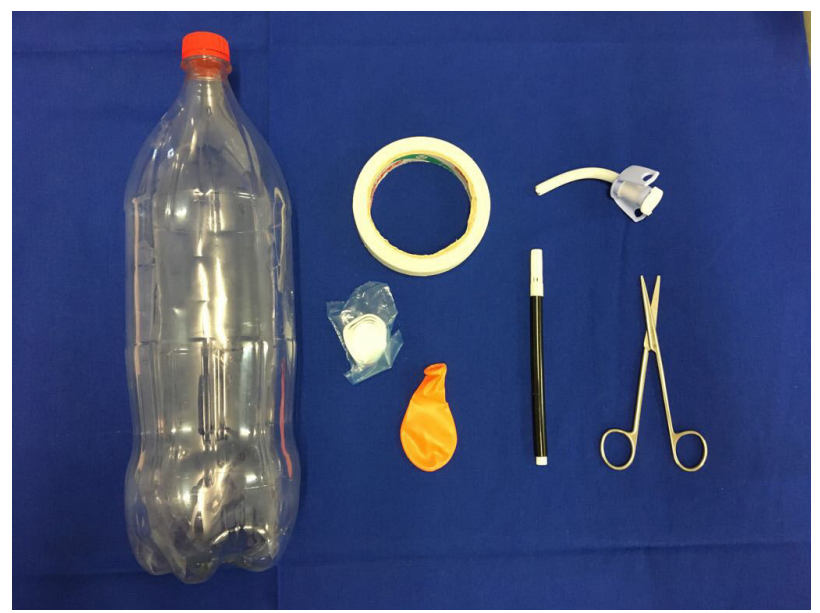

Figura 1. Exemplos de materiais usados na confecção do modelo artificial de traqueostomia e cricotireoidostomia

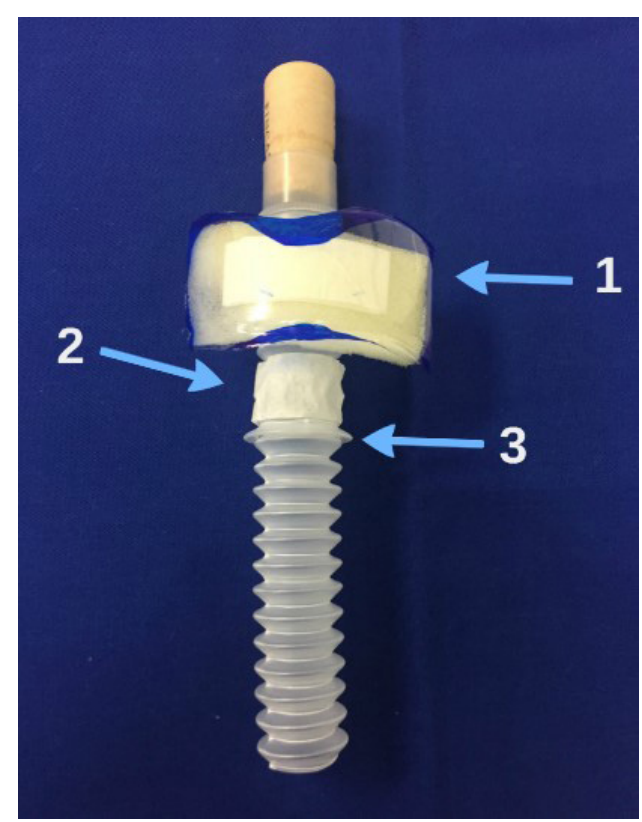

Figura 2. Traqueia sanfonada com sua extremidade superior fechada por uma rolha: 1 . Tira de plástico de garrafa pet simulando a cartilagem tireoidea; 2 . Traqueia com abertura envolta com fita crepe simulando a membrana cricotireoidea; 3 . Anel de traqueia simulando cartilagem cricoidea

\section{RESULTADOS}

O experimento resultou em um modelo de cricotireoidostomia e traqueostomia, reutilizável até quatro vezes em um único modelo, de aproximadamente cinco reais de custo, podendo ser confeccionado em 15 minutos.

Trinta e sete alunos que demonstraram interesse em participar, de forma voluntária, já haviam realizado o procedimento em modelo animal, após uma aula habitual da grade curricular sobre manejo de vias aérea. A maioria (54\%) era do sexo feminino, 64,8\% tinham interesse em

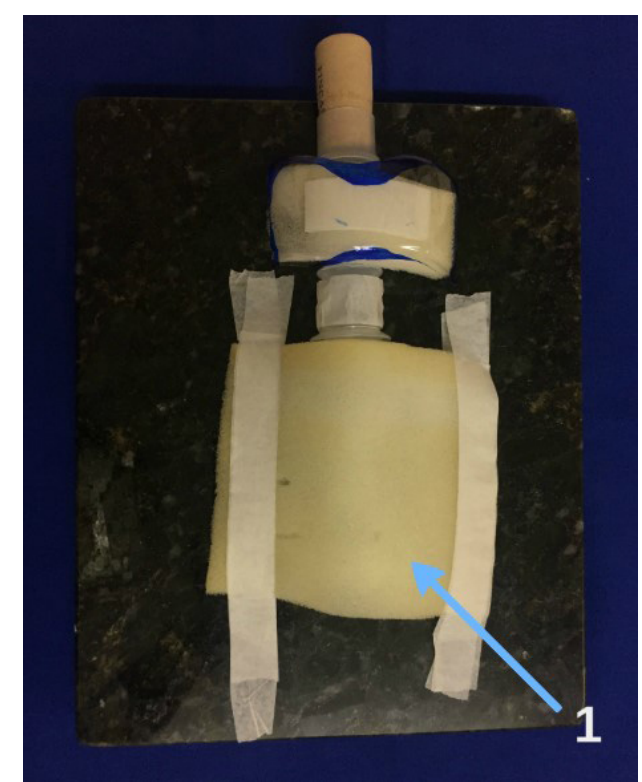

Figura 3. 1. Espuma simulando músculos pré-laríngeos

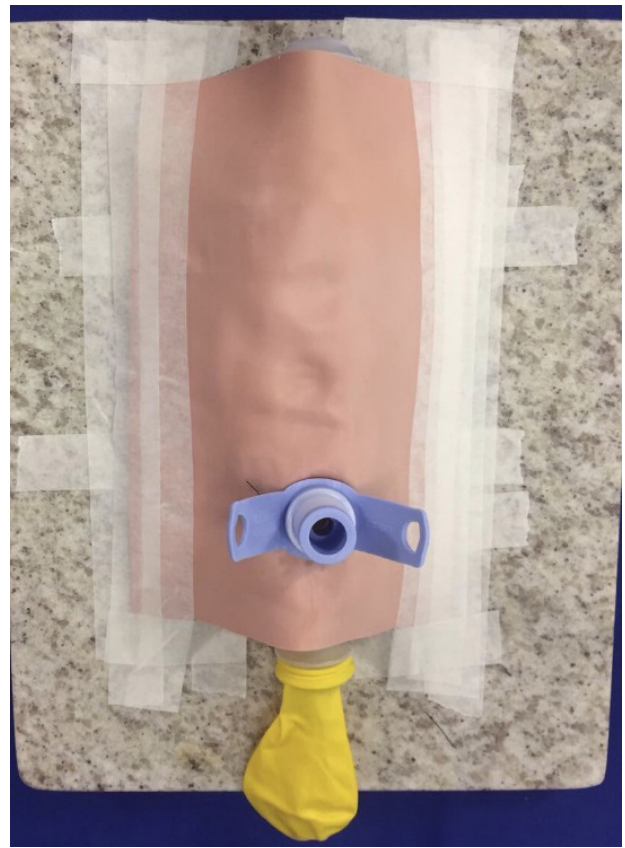

Figura 4. Modelo final, com a pele artificial envolvendo toda a estrutura e testada com a passagem da cânula de traqueostomia, após a realização do procedimento

área cirúrgica, $62,2 \%$ tinham entre 20 e 23 anos, 24,3\% entre 24 e 26 anos, $13.5 \%$ maiores de 26 anos, 83,8\% cursavam o $7^{\circ}$ período, $5,4 \%$ cursavam o $10^{\circ}$ período e $10,8 \%$, o $11^{\circ}$.

Em relação ao questionário, pudemos observar que as críticas em relação às dificuldades encontradas foram quanto ao tipo de material utilizado para a confecção. Quarenta por cento dos alunos disseram que o modelo não se assemelhava ao material biológico, como, por exemplo, a maleabilidade do material que simula a traqueia, o que 
poderia até trazer prejuízo na técnica do procedimento, (Gráfico 1). Porém, 73\% dos alunos afirmaram que o modelo sintético é necessário para a graduação, sendo que a maior vantagem encontrada seria a possibilidade de prática em momentos posteriores a aula habitual, além de reutilização do mesmo modelo mais de uma vez e a minimização do uso de peças animais em laboratórios (Gráfico 2). Além disso, mais de 70\% dos alunos sugeriram que deveria haver a complementação da graduação com as peças sintéticas e $27 \%$ acreditam que apenas o material biológico deveria ser o utilizado (Gráfico 3).

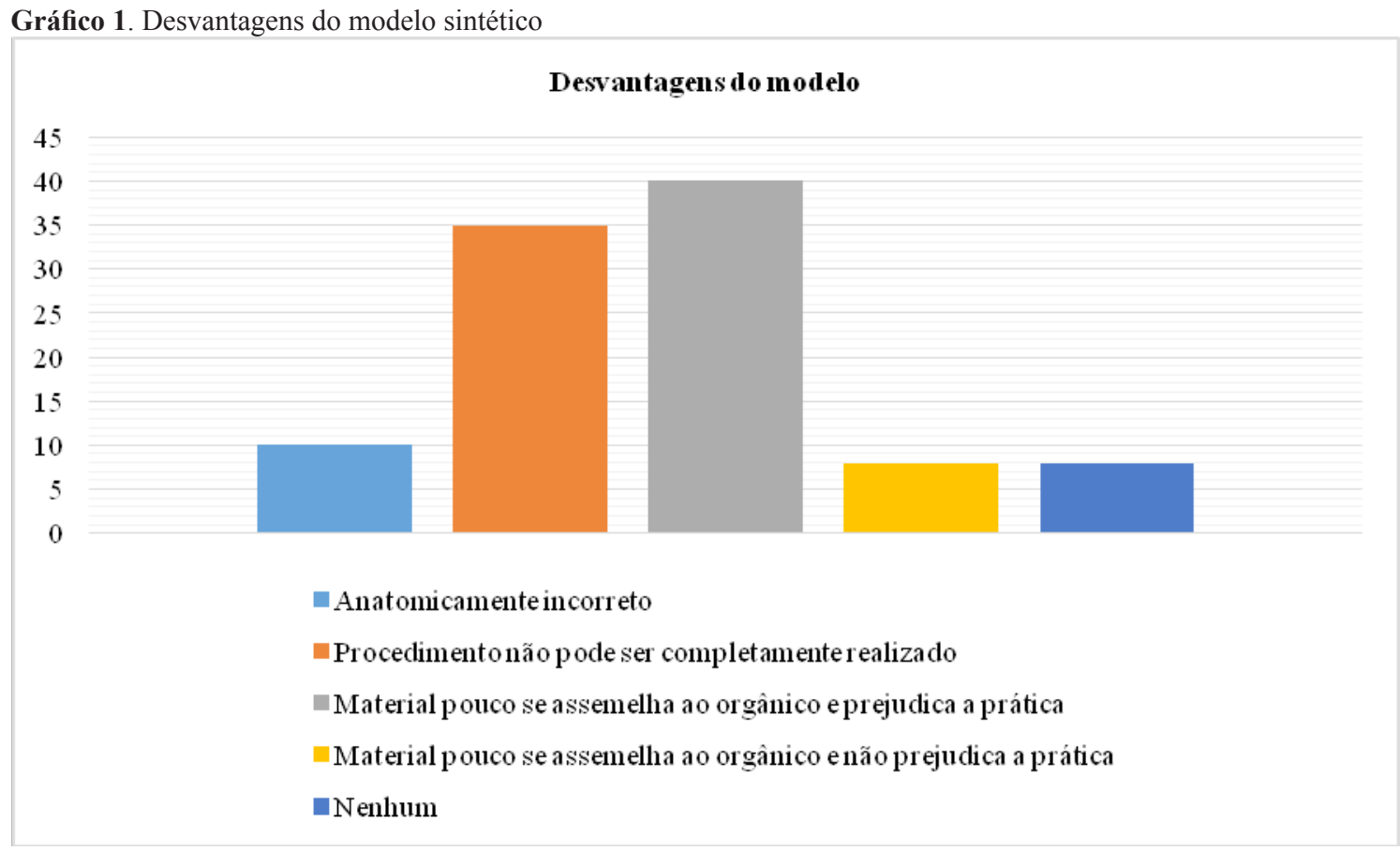

Gráfico 2. Vantagens do modelo sintético

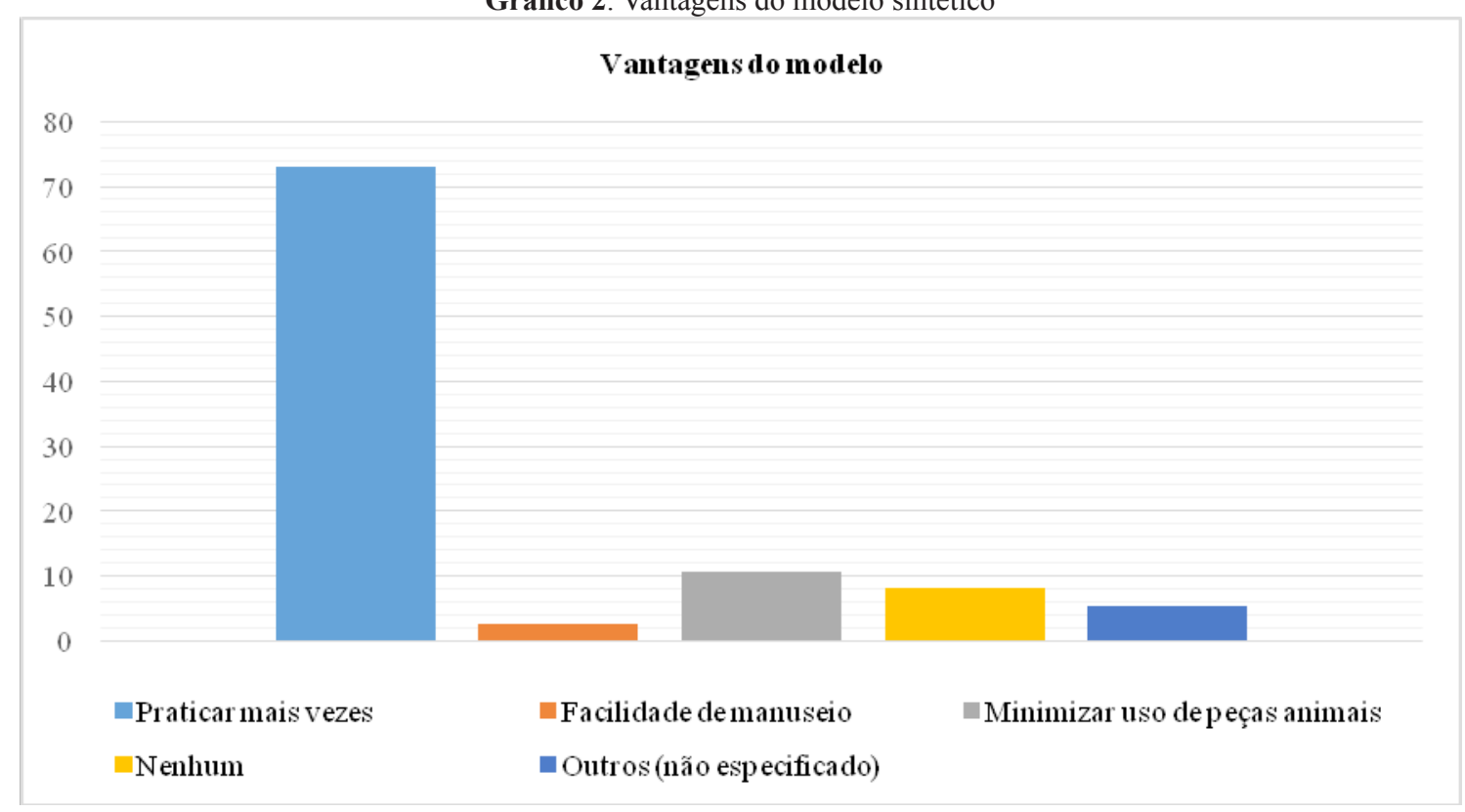


Gráfico 3. Preferências de materiais para ensino e treinamento de habilidades cirúrgicas

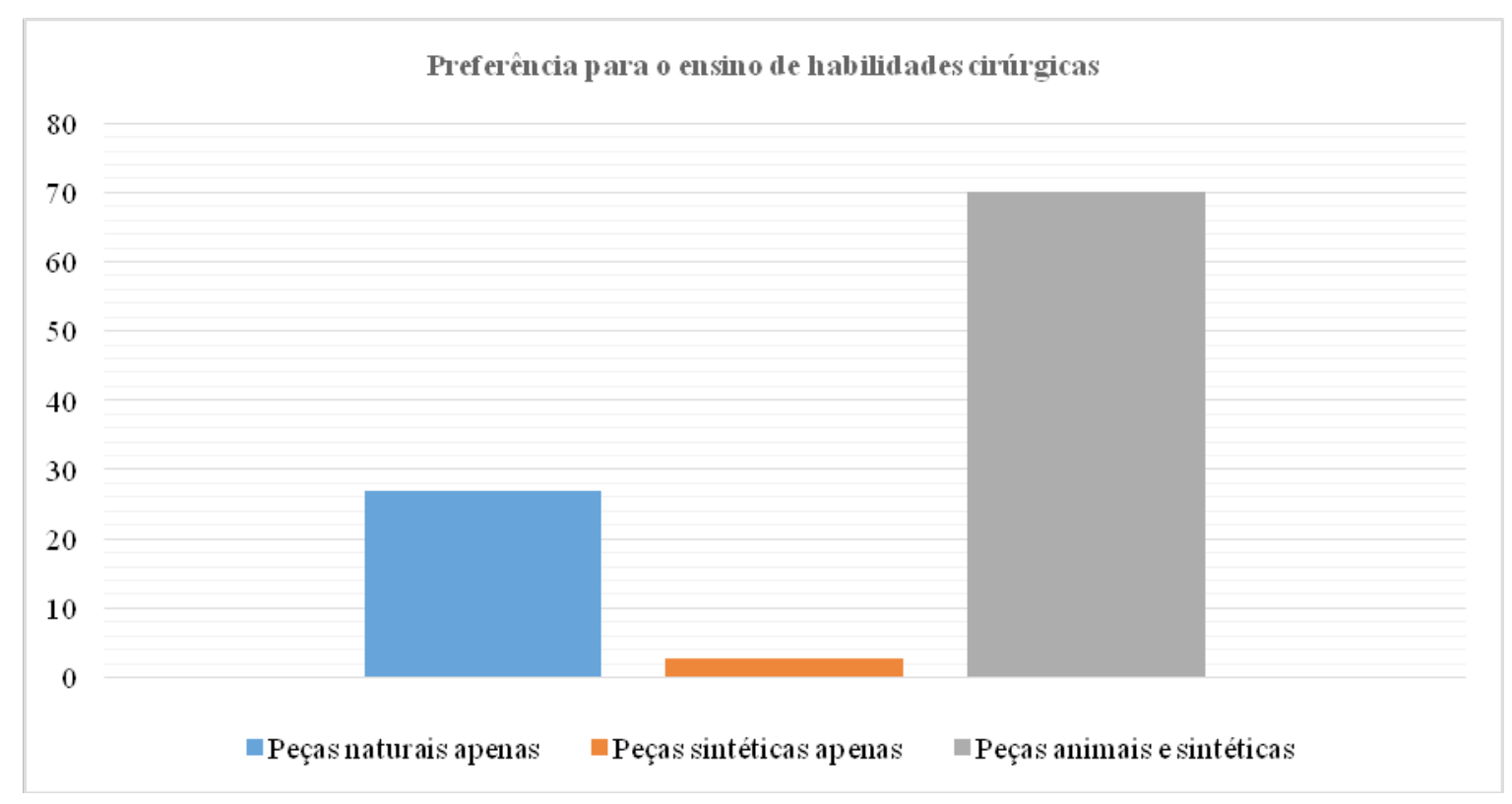

\section{DISCUSSÃO}

Este modelo mostrou-se tratar de uma alternativa de baixo custo que respeita os princípios da bioética, de fácil reprodutibilidade e muito útil em comparação aos modelos animais, para o treinamento de técnica e habilidade e, consequentemente, diminuição de iatrogenias. De fato, ter a habilidade e confiança para realizar o procedimento, que pode ser tanto pré-hospitalar quanto intra-hospitalar, é de extrema importância para o médico generalista e para o cirurgião ${ }^{5}$.

$\mathrm{O}$ material de treinamento biológico tem sua vantagem pela prática em tecido verdadeiro ${ }^{6,7,8}$, mas tambéem tem desvantagens, como sua pequena durabilidade, dificuldade de armazenamento, alto custo, não ser reutilizável e o fato de as novas normas das sociedades protetoras dos animais estarem cada vez mais rigorosas ${ }^{1}$. Por outro lado, com os materiais sintéticos que temos disponíveis, podemos ter uma estrutura bastante próxima da realidade, tanto em relação à anatomia quanto em relação à textura e à localização das estruturas.

Existem outros modelos sintéticos, como o desenvolvido pela Southern Illnois University School of Medicine, que criou um modelo para cricotireoidostomia, com materiais também de baixo custo e reutilizável ${ }^{9}$. Nosso modelo, diferentemente, visa a utilização de um dispositivo para treinamento dos dois procedimentos: a traqueostomia e a cricotireoidostomia.

Analisando o modelo in vivo e as peças biológicas, encontramos dificuldades com relação ao custo para anestesia, ao pré e pós-operatório dos animais e o valor elevado de peça para uma única realização de procedimentos, devido ao descarte imediato após a prática, enquanto, no modelo sintético apresentado neste artigo, obtivemos um baixo custo e reutilização em até quatro vezes com o mesmo material.

Ficou evidente o quanto as opiniões divergem sobre a substituição do modelo biológico pelo sintético, entretanto pode-se observar como a realização dos procedimentos no modelo sintético não fez com que os alunos apresentassem grandes dificuldades no aprendizado e efetuação da técnica e muito menos no reconhecimento das estruturas que simulariam a anatomia, todas devidamente identificadas por todos os participantes.

Com este trabalho, pode-se observar o quanto a resistência à substituição do modelo sintético ainda está fortemente presente ${ }^{3,8}$. Por meio dos resultados obtidos neste estudo, percebe-se que a substituição completa não é a melhor solução, e sim uma excelente opção para complementar o ensino dos procedimentos.

Sabe-se que modelos sintéticos não substituem modelos in vivo ou biológico, porém, com a sua utilização, veem-se inúmeros pontos positivos comparados aos modelos usados no ensino tradicional. O modelo sintético recebeu opiniões positivas, alegando maior facilidade de entendimento da técnica cirurgica e do passo a passo do procedimento, mesmo sendo alvo de críticas por não ser fidedigno em relação à textura, especificamente quanto ao material da traqueia, que se mostrou mais rígido que o esperado, e à anatomia. O modelo também se sobressai por treinar exaustivamente a técnica do aluno iniciante em habilidades cirúrgicas antes de usar o modelo animal, 
poupando gastos e não usando animais de forma banal e sem restrição. $\mathrm{O}$ modelo em questão segue as novas linhas de ensino, como do aluno pró-ativo, que busca conhecimento. Por fim, apontando os principais pontos do modelo, este mostrou-se um bom investimento para os devidos fins de aprendizagem, desde os alunos iniciantes aos mais graduados, embora não seja um modelo adequado para substituição.

\section{CONCLUSÃO}

Este modelo apresenta-se como uma alternativa, de baixo custo, reprodutível e de boa aceitação entre os acadêmicos para complementar o ensino de técnicas cirúrgicas nos procedimentos de traqueostomia e cricotireoidostomia. $\mathrm{O}$ intuito é estimular o desenvolvimento de habilidade, confiança e técnica, para que o futuro médico, tanto generalista quanto cirurgião, se sinta preparado para realizar o procedimento com sucesso. Saliente-se, contudo, que esse tipo de modalidade não deve substituir o aprendizado em peças anatômicas e em ambulatórios médicos, e espera-se que as dificuldades técnicas de material, por exemplo, não prejudiquem o aprendizado do acadêmico.

Agradecimentos: Gostaríamos de agradecer a Profa. Dra. Patricia Maluf Cury, Profa. Dra. Carolina Colombelli Pacca e Profa. Norma Barbosa Novaes por não medirem esforços em ajudar na revisão deste artigo e ao Dr. Toufic Anbar Neto e à FACERES por serem estimuladores e entusiastas dos alunos na pesquisa científica.

Participação dos autores no artigo: Natássia Alberici Anselmo - Desenvolvimento dos modelos, levantamento bibliográfico, aplicação do questionário, análise e estatísticas dos resultados, redação do texto e correspondente responsável junto aos editores; Kênia Maria Jordão Cazon, Amely Covalero da Silva Pinto, Antonio Carlos Morale Guerra Júnior: Desenvolvimento dos modelos, levantamento bibliográfico, aplicação do questionário, análise e estatísticas dos resultados e redação do texto; Carlos Dario da Silva Costa e Raphael Raphe: Idealizador e revisor do artigo.

\section{REFERÊNCIAS}

1. Diniz R, Duarte ALA, Oliveira CAS, Romiti M. Animais em aulas práticas: podemos substituí-los com a mesma qualidade de ensino? Rev Bras Educ Med. 2006;30(2):31-40. http:// dx.doi.org/10.1590/S0100-55022006000200005.

2. Ricz HMA, Freitas LCC, Mamede RCM. Traqueostomia. Medicina (Ribeirão Preto). 2011;44(1):63-9. http://dx.doi. org/10.11606/issn.2176-7262.v44i1p63-69.

3. Demirel D, Butler KL, Halic T, Sankaranarayanan G, Spindler D, Cao C, et al. A hierarchical task analysis of cricothyroidotomy procedure for a virtual airway skills trainer simulator. Am J Surg. 2016;212(3):475-84. https:// doi.org/10.1016/j.amjsurg.2015.08.029.

4. Denadai R, Souto LRM. Organic bench model to complement the teaching and learning on basic surgical skills. Acta Cir Bras. 2012;27(1):88-94. http://dx.doi.org/10.1590/S010286502012000100015 .

5. Couto RS, Veloso AC, Antunes FG, Ferrari R, Carneiro RGF. Device model for training of laparoscopic surgical skills. Rev Col Bras Cir. 2015;42(6):418-20. http://dx.doi. org/10.1590/0100-69912015006012
6. Ferreira LM, Hochman B, Barbosa MVJ. Experimental models in research. Acta Cir Bras. 2005;20(2):28-34. http:// dx.doi.org/10.1590/S0102-86502005000800008.

7. King W, Teare J, Vandrevala T, Cartwright S, Mahammed KB, Patel B. Evaluation of a novel Surgicric cricothyroidotomy device for emergency tracheal access in a porcine model. Anaesthesia. 2016;71(2):177-84. https://doi.org/10.1111/ anae. 13275 .

8. Takayesu JK, Peak D, Stearns D. Cadaver-based training is superior to simulation training for cricothyrotomy and tube thoracostomy. Intern Emerg Med. 2017;12(1):99-102. https:// doi.org/10.1007/s11739-016-1439-1.

9. Gierek T, Majzel K, Markowski J, Iwanowski P, Szewczyk P. Plastic tracheostomy tube of Luer's type. Otolaryngol Pol. 1998;52(4):499-501.

Recebido em: 31.03 .17

Aceito em: 12.02 .18 